MATHEMATICS OF COMPUTATION

Volume 78, Number 266, April 2009, Pages 1197-1199

S $0025-5718(08) 02170-4$

Article electronically published on August 1, 2008

\title{
TEN NEW PRIMITIVE BINARY TRINOMIALS
}

\author{
RICHARD P. BRENT AND PAUL ZIMMERMANN
}

\begin{abstract}
We exhibit ten new primitive trinomials over $\mathrm{GF}(2)$ of record degrees $24036583,25964951,30402457$, and 32582657 . This completes the search for the currently known Mersenne prime exponents.
\end{abstract}

Primitive trinomials of degree up to 6972593 were previously known 4 . We have completed a search for all known Mersenne prime exponents [7. Ten new primitive trinomials were found. Our results are summarized in the following theorem:

Theorem 1. For the integers $r$ listed in Table 1, the primitive trinomials $x^{r}+x^{s}+1$ of degree $r$ over $\mathrm{GF}(2)$ are exactly those given in Table 1, and the corresponding reciprocal trinomials $x^{r}+x^{r-s}+1$.

Proof. From the GIMPS Project [7, the integers $r$ listed in Table 1 are exponents of Mersenne primes $2^{r}-1$. Thus, irreducible trinomials of degree $r$ are necessarily primitive. Irreducibility of the trinomials listed in Table1 1 follows from the authors' computations, using the new algorithm described in [5, 6] (verified using the algorithm of [3] and independently verified by Allan Steel using Magma). Finally, the fact that no irreducible trinomials were missed during the search, for those degrees $r$, follows from the certificates given on the authors' web pages [1].

Remarks. The integers $r$ listed in Table 1 are the known Mersenne exponents of the form $r= \pm 1 \bmod 8$ in the interval [100000,32582657]. For smaller exponents, omitted to save space, see [10] or our web site [1. According to the GIMPS Project [7, the list is complete for $r \leq 16300000$. Known Mersenne exponents of the form $r= \pm 3 \bmod 8$ for $r>5$ cannot be the degrees of irreducible trinomials due to Swan's theorem [12] the possibility $x^{r}+x^{2}+1$ permitted by Swan's theorem is easily ruled out in all known cases with $r>5$; see the authors' web site 11.

Our search used a new algorithm [5, 6] relying on fast arithmetic in $\operatorname{GF}(2)[x]$, whose details are given in 2. Another significant improvement over previous work is that certificates were produced; this enables one to check easily that the claimed nonprimitive trinomials are indeed reducible. A certificate is simply an encoding of a nontrivial factor of smallest degree. A $2.4 \mathrm{Ghz}$ Intel Core 2 takes only 15 minutes to check the certificates of all 16291325 reducible trinomials $(s \leq r / 2)$ of degree $r=32582657$ with our check-ntl program based on NTL [11.

Received by the editor April 15, 2008.

2000 Mathematics Subject Classification. Primary 11B83, 11Y16; Secondary 11-04, 11T06, $11 \mathrm{Y} 55,12-04$

Key words and phrases. $\mathrm{GF}(2)[x]$, irreducible polynomials, irreducible trinomials, primitive polynomials, primitive trinomials, Mersenne exponents, Mersenne numbers.

(C)2008 American Mathematical Society Reverts to public domain 28 years from publication 
TABLE 1 . Known primitive trinomials $x^{r}+x^{s}+1$ whose degree is a Mersenne exponent $r \geq 100000$, for $s \leq r / 2$.

\begin{tabular}{|r|c|l|}
\hline$r$ & $s$ & \multicolumn{1}{|c|}{ Notes } \\
\hline 110503 & 25230,53719 & Heringa et al. [8] \\
132049 & $7000,33912,41469,52549,54454$ & Heringa et al. [8] \\
756839 & $215747,267428,279695$ & Brent et al. [3] \\
859433 & 170340,288477 & Brent et al. [3]. Kumada et al. [9] \\
3021377 & 361604,1010202 & Brent et al. [3] \\
6972593 & 3037958 & Brent et al. [4] \\
24036583 & 8412642,8785528 & Brent and Zimmermann, 2007 \\
25964951 & $880890,4627670,4830131,6383880$ & Brent and Zimmermann, 2007 \\
30402457 & 2162059 & Brent and Zimmermann, 2007 \\
32582657 & $5110722,5552421,7545455$ & Brent and Zimmermann, 2008 \\
\hline
\end{tabular}

\section{ACKNOWLEDGEMENTS}

The authors thank Allan Steel, who independently verified (with Magma) the ten new primitive trinomials, and the authors of the Magma and NTL software tools that were used to check reducibility of the other trinomials. Part of the computations reported in this paper were carried out using the Grid'5000 experimental testbed, an initiative of the French Ministry of Research through the ACI GRID incentive action, INRIA, CNRS and RENATER and other contributing partners (see https://www.grid5000.fr). The work of the first author was supported by the Australian Research Council.

\section{REFERENCES}

[1] Richard P. Brent, Search for primitive trinomials (mod 2), http://wwwmaths.anu.edu.au/ ^brent/trinom.html, 2008.

[2] Richard Brent, Pierrick Gaudry, Emmanuel Thomé, and Paul Zimmermann, Faster multiplication in $\mathrm{GF}(2)[x]$, Proc. of the 8th International Symposium on Algorithmic Number Theory (ANTS VIII), Lecture Notes in Computer Science 5011, Springer-Verlag, 2008, 153-166.

[3] Richard P. Brent, Samuli Larvala, and Paul Zimmermann, A fast algorithm for testing reducibility of trinomials mod 2 and some new primitive trinomials of degree 3021377, Math. Comp. 72 (2003), 1443-1452. MR1972745 (2004b:11161)

[4] _ A primitive trinomial of degree 6972593, Math. Comp. 74 (2005), 1001-1002. MR2114660 (2005h:11054)

[5] Richard P. Brent and Paul Zimmermann, A multi-level blocking distinct degree factorization algorithm (extended abstract), Proceedings of the 8th International Conference on Finite Fields and Applications (Fq8) (Melbourne, Australia), 2007.

[6] _ A multi-level blocking distinct degree factorization algorithm, Contemporary Mathematics, special issue, to appear. Also available as arXiv:0710.4410.

[7] The Great Internet Mersenne Prime Search, http://mersenne.org/

[8] J. R. Heringa, H. W. J. Blöte, and A. Compagner, New primitive trinomials of Mersenneexponent degrees for random-number generation, International Journal of Modern Physics C 3 (1992), 561-564. MR 1169571 (94a:11118)

[9] T. Kumada, H. Leeb, Y. Kurita, and M. Matsumoto, New primitive $t$-nomials $(t=3,5)$ over GF(2) whose degree is a Mersenne exponent, Math. Comp. 69 (2000), 811-814; MR.1665959 (2000i:11183) corrigenda: ibid 71 (2002), 1337-1338; MR1898761 (2003c:11153)

[10] Y. Kurita and M. Matsumoto, Primitive $t$-nomials $(t=3,5)$ over $\mathrm{GF}(2)$ whose degree is a Mersenne exponent $\leq 44497$, Math. Comp. 56 (1991), 817-821. MR1068813(91h:11138) 
[11] Victor Shoup, NTL: A library for doing number theory, http://www.shoup.net/ntl/, 2007.

[12] R. G. Swan, Factorization of polynomials over finite fields, Pacific J. Math. 12 (1962), 10991106. MR0144891(26:2432)

Australian National University, Canberra, Australia

E-mail address: trinomials@rpbrent.com

INRIA NANCy, Grand Est, Villers-LÈs-NANCy, France

E-mail address: Paul.Zimmermann@loria.fr 